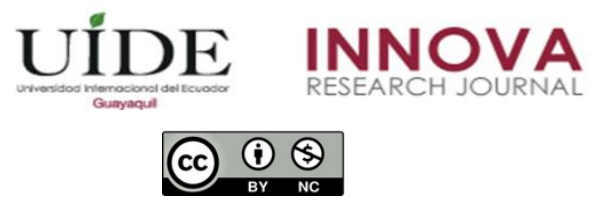

INNOVA Research Journal, ISSN 2477-9024

(Enero-Abril 2020). Vol. 5, No.1 pp. 31-46

DOI: https://doi.org/10.33890/innova.v5.n1.2020.1120

URL: http://revistas.uide.edu.ec/index.php/innova/index

Correo: innova@uide.edu.ec

\title{
El uso de las TIC en el aprendizaje en la Universidad caso UTMACH
}

\section{The use of ICT in learning at the UTMACH case UTMACH}

Patricia Alexandra Uriguen Aguirre

https://orcid.org/0000-0003-3095-8765

Flor Yelena Vega Jaramillo

Ángel Eduardo Luna-Romero

Universidad Técnica de Machala, Ecuador

Autor por correspondencia: puriguen@utmachala.edu.ec; fvega@utmachala.edu.ec; aeluna@utmachala.edu.ec

Fecha de recepción: 22 de septiembre del 2019 - Fecha de aceptación: 05 de diciembre del 2019

\section{Resumen}

El presente estudio se estableció de la necesidad de realizar un análisis comparativo de diversas percepciones de los estudiantes universitarios acerca del tema relacionado con el empleo de las tecnologías de investigación y comunicación en la educación, puesto que se puede inferir que el empleo de estas herramientas tecnológicas permiten un mejor desarrollo de la enseñanza académica en el área económica; propiciando de esta manera el interés de los estudiantes al emplear nuevas metodologías de instrucción y un ambiente educativo factible, mediante la dinamización de las clases impartidas, consolidando el conocimiento de los estudiantes, con lo impartido en el aula de clase. Se determina el desarrollo de esta investigación que se llevó a cabo mediante recopilación de diversos temas relacionados dirigido a los estudiantes universitarios. Por consiguiente, se procedió a la aplicación de encuestas a diversos estudiantes de la Universidad Técnica de Machala. Los resultados de la investigación demuestran que el conocimiento, uso e interés de las TIC en los estudiantes de la carrera de economía se llegan a convertir en una de las estrategias fundamentales al momento de efectuar una determinada actividad académica, influyendo en la adquisición de los conocimientos netamente estructurados. Palabras clave: economía; TIC; aprendizaje; enseñanza; universidad

\footnotetext{
Abstract

The present study measures the need to perform a comparative analysis of various perceptions of university students on the subject related to the use of research and communication technologies in education, since the use of these technological tools can be inferred allows a better development of academic teaching in the economic area; thus promoting the interest of students by using new instructional methodologies and a feasible educational environment, by revitalizing the classes taught, consolidating the students' knowledge, with what is taught in the classroom. The development of this research that was carried out by compiling various topics related to university students is determined. By evaluation, the application of surveys to various students of the Technical University of Machala is being processed. The results of the research show that the knowledge, use and interest of ICT in students of the economics career become one of the 
fundamental strategies at the time of carrying out a certain academic activity, influencing the acquisition of knowledge clearly structured.

Key words: Economy; TIC'S; learning; teaching; university

\section{Introducción}

En la actualidad el uso y la interacción de las personas con las tecnologías de la información y la comunicación (TIC) han generado un impacto y posicionamiento en el ámbito económico, político, social educativo y cultural permitiendo dar paso a la sociedad del conocimiento cuya característica esencial es generar su propio beneficio, (Ruiz-Jaramillo \& Vargas-Yáñez, 2018). La sociedad en general se ha visto impactada por el desarrollo abarcando aspectos y actividades relevantes como la educación salud y producción construyéndose así una nueva forma de asumir retos y mejorarlos mediante la generalización del uso de las nuevas tecnologías. La aplicación y uso de TIC en el aula de clase facilita al docente y estudiante de herramientas tecnológicas, dándole protagonismos al docente en el aprendizaje (Fernández-Cruz, Fernández-Díaz, \& Rodríguez-Mantilla, 2018). Proporcionando una renovación didáctica en el aprendizaje en la que se ubica una metodología activa e innovadora para el estudiante.

Las tecnologías de información y comunicación se constituyen como un conjunto de herramientas e instrumentos tecnológicos que facilitan a las personas adquirir conocimientos y habilidades para afrontar situaciones de la vida diaria, en las universidades permite expandir su investigación. en nuevas áreas, fortalecer sus lazos con la sociedad (Quiroga-Parra, TorrentSellens, \& Murcia-Zorrilla, 2017). En lo concerniente al ámbito educativo el empleo de las TIC permite emplear estrategias métodos y recursos necesarios para potenciar y enriquecer el conocimiento (Plaza de la Hoz, 2016). Por lo que incluir el uso de las TIC en áreas educativas es primordial; asumir nuevos roles en el contexto para fortalecer el vínculo de enseñanzaaprendizaje tomando como sujetos principales a los estudiantes y profesores, ha sido fuerte y transversal en múltiples contextos sin embargo se confronta que las nuevas tecnologías no fueron concebidas ni imaginadas para la educación; es decir no aparecen naturalmente en los sistemas educacionales, y ocasionalmente su introducción respondió a intencionalidades educativas.

Involucrarse en un proceso de enseñanza-aprendizaje donde se implementan TIC, les permite apropiarse de su uso, pues les facilita participar de forma activa en la sociedad siendo capaces de demostrar nuevas habilidades y competencias de manera más creativas (Escardíbul, 2016). No únicamente pueden emplearse como simples herramientas de trabajo en el aula de clase, pues además estás constituyen nuevas técnicas, modalidades conversaciones, formas de construir identidades, actitudes, aptitudes y vínculos en el proceso (Bordas-Beltrán \& ArrasVota, 2018); Tanto profesores como estudiantes de los distintos niveles y áreas en la educación disponen y hacen uso de las innovaciones con las que cuentan y que al introducir las tecnologías de la información y comunicación dentro de este ámbito, la perspectiva en cuanto a la utilización de estos recursos cambia de manera drástica principalmente al combinar recursos físicos como dinámicos, generando de esta forma un indudable valor añadido en la educación y aprendizaje (Vivanco, 2015). Por lo que diferentes procesos, así como estrategias de enseñanza y aprendizaje en cada una de las asignaturas deben estar en continuo mejoramiento y de manera exclusiva la economía que es lo que analizaremos en el presente artículo. 
Los profesores deben de tomar las capacitaciones necesarias para incentivar, desarrollar junto con sus aprendices técnicos de mejoramiento que incentiven al estudiante a colmar de conocimientos, por ende en necesario y fundamental crear o hacer de estas herramientas su utilización dinámica (Sefo, Granados, Lazaro, \& Fernández-Larragueta, 2017), En referencia a la formación de los profesores en el conocimiento que contengan las bases necesarias para fomentar una educación de calidad y aportar de forma positiva a los jóvenes que en un futuro serán quienes contribuyan al crecimiento económico, político, social y cultural de un país (Duarte, 2007). En ciertos aspectos el uso de la tecnología es positivo y ayuda a la formación profesional de cada individuo de una sociedad, por ello el docente debe saber inculcar a sus estudiantes un manejo apropiado para obtener beneficios deseados.

Desde que el ser humano tuvo la necesidad de aprender a desarrollar sus capacidades para poder subsistir se ve en la obligación de educarse siendo está considerada por mucho tiempo como eslabón privilegiado que articula la integración cultural, la movilidad social y el desarrollo productivo (Unesco, 2013). Considerándose a las universidades como el lugar del más alto nivel de aprendizaje y hogar de la ciencia e innovación (Altbach, 2011). Siendo indispensable la educación para la formación y el desarrollo de las personas en la sociedad. Es evidente el enorme impacto de la información y de las tecnologías de la información y la comunicación en los distintos aspectos, contextos sociales y en el vivir diario de cada individuo.

En la enseñanza de economía, las tecnologías de la información y la comunicación cumplen un papel importante (Cárdenas, 2012), en el estudio de la economía el manejo y actualización de la información es de gran importancia con el uso de internet, intranet de la universidad y de otras universidades correo electrónico personal e institucional, uso y acceso a las TIC en computadoras personales teléfono inteligentes, dispositivos electrónicos en este sentido junto con la aplicación de programas estadísticos, software como Microsoft Excel, Google Spreadsheet, SPSS (SPSS Statistics Base), Minitab, Statgraphics, R The R Project for Statistical Computing, EViews, los estudiantes pueden analizar bases de datos, actualizar y acceder a información muy diversa que contribuye al estudio y análisis de la economía.

Se considera a las tecnologías de la información y comunicación como elemento importante, estratégico e innovador en la formación del conocimiento (Ortega, 2011). Por lo que se efectúa dentro de todas las dimensiones culturales, proliferando el uso de nuevas tecnologías en beneficio a la educación, mejorando el ecosistema comunicacional. Esto se forma en un contexto de gran relevancia cuando de problemas educacionales se trata, tomando en cuenta que gracias a la implementación de estas tecnologías se generan un escenario que los estudiantes y profesores intercambian configuraciones mentales, emotivas y sensoriales (Plaza de la Hoz, 2016). Forjando un cambio dentro de las formas de interactuar, relacionarse y de comunicación, estos vínculos entre la tecnología y la educación deben generar un avance tecnológico que permita desarrollar a la forma de aprendizaje dando satisfacción en las necesidades propias de la educación moderna.

América Latina ha tenido una utilización de forma pausada, apostando a la incorporación de las tecnologías de la información y comunicación (Bejar, 2007). Se considera a estas como trascendentales para competir en el mundo educativo, el uso de las TIC se ha convertido en los últimos años en un instrumento de apoyo para la educación, para el plano micro - analítico que 
mejora el desempeño en el aula, como en la parte macro - analítico en la evaluación de distinto programas y sistemas, (Jornet, González-Such, \& García-Bellido, 2012). Otros sectores que no tienen relación de forma directa con la educación fueron los primeros en implementar la utilización de la tecnología en sus inicios, tomando en cuenta que los primeros computadores personales (PC) llegaron a las empresas y no a los establecimientos educativos proceso de uso y aplicación en la población fue lento.

Las estadísticas muestran de su verdadera importancia dentro de la educación y del sector productivo se observó después de la década de los años 2000. Algunos países latinoamericanos llevan proyectos de incorporación de las TIC en la educación orientadas al modelo 1x1, el Plan Ceibal en Uruguay, Laptop por niño en Perú, Conectar Igualdad en Argentina y uso de tecnología portátil 1 a 1 en aula desarrollada en Chile (Lagos \& Silva, 2011). Ecuador no es ajeno a las tendencias de las tecnologías de la información, en el campo del conocimiento en las instituciones de educación superior mejorando la inclusión social.

\section{Importancia del Problema}

La educación superior en la sociedad actual ya no es considerada solamente como un instrumento para promover la cultura de las personas y la forma de socializar dentro de su entorno social (Luna-Romero, Vega, \& Carvajal, 2018). Podemos notar que la utilización correcta de la tecnología se ha convertido en una necesidad prioritaria estratégica a nivel mundial (Sánchez \& González, 2014). Si tomamos en consideración que dentro de la sociedad del conocimiento necesita de estructuras flexibles (entornos virtuales de aprendizaje) que doten a los estudiantes universitarios de un amplio acceso al conocimiento, esto ayudara a que exista una capacitación personal crítica que ayude a la interpretación de la información y la concepción del propio conocimiento, logrando el desarrollo de nuevas metodologías donde las tecnologías de información y comunicación tengan un rol determinante, y sirvan como recursos de apoyo que permitirían promover el aprendizaje y generar nuevos espacios educativos (Rivero, Chávez, Vásquez, \& Blumen, 2016).

En las universidades ecuatoriana las tecnologías de la información y comunicación tienden a ser presentadas como generadoras del cambio, e innovación didáctica (Peñaherrera, 2011). En primer lugar, podemos mencionar que las que se denominan nuevas tecnologías y las tradicionales, desde su concepción se dieron fuera del contexto educativo y después por la importancia que las mismas presentaron se dio la necesidad de incorporarlas; en segundo lugar, dentro de la educación este fundamentalismo tecnológico que algunas veces nos rodea, en principio se ha otorgado a la tecnología y después se ha elaborado el problema académicos que por medio de las tecnologías de la información y comunicación tendría una solución en lo educativo.

Las TIC en la educación brindan un conjunto de servicios informáticos que son utilizados a través de una red de informática y ordenadores, el uso de estas tecnologías en el ámbito educativo tiene como objetivo facilitar y obtener información avanzada permitiendo que el estudiante pueda investigar, sintetizar, analizar y llegar a obtener sus propias conclusiones (Tejedor \& García-Valcárcel, 2006). En el audio conferencias es indispensable ya que permite a un grupo determinado de personas que se encuentren distante tengan la oportunidad de 
comunicarse con otras personas para dialogar diferentes asuntos, por lo que esto se convierte en el intercambio de experiencias para el aprendizaje; en los espacios de web, nubes o drives educativos con el uso de internet permite a los usuarios poder almacenar información, videos, imágenes que estén disponibles para la red permitiendo seguridad facilidad y flexibilidad con diferente calidad de información (López, Cerveró, Rodríguez, Félix, \& Esteban, 2013). Los correos electrónicos una de las actividades más frecuente utilizadas en la sociedad y en especial en el sistema educativo debido a su sistema de mensajería para poder enviar y recibir archivos de una manera muy rápida (Nieto \& Rodriguez, 2007); en vista de estos desarrollos, las universidades necesitan encontrar su propia posición para la integración de saberes con un impacto positivo en la enseñanza con acceso a distintos recursos de TIC.

Argumentamos que la enseñanza y el aprendizaje efectivos con las TIC implican que los docentes encuentren formas de construir puentes entre el aprendizaje individual e idiosincrásico y el previsto (Campos \& Navarro, 2017). De esta manera, ¿cómo podemos aprender a usar una nueva tecnología de una manera transformadora? La lectura de artículos que hablan sobre el potencial de las TIC para aprender no es suficiente a menos que esta lectura esté integrada en la experimentación práctica. No es probable que la experimentación práctica sea suficiente a menos que esté integrada en la práctica general, involucrando tecnología de la información y comunicación trabajando de manera distinto compartiendo el conocimiento, la experiencia y los conocimientos relacionados con el trabajo (Lera \& Blanco, 2003).

En el Ecuador las políticas públicas con respecto a la educación superior hace énfasis principalmente en generar una construcción del conocimiento, tomando en cuenta la necesidad tecnológica dentro del campo laboral, las universidades dentro del Ecuador optan por implementar las TIC, aunque este hecho no es del todo eficiente, esta implementación tecnológica implica un cambio, una transformación a fondo del proceso educativo desechando de forma permanente las recurrentes práctica de ver a los profesores como un pedagogo que lo sabe todo y que imparte el conocimiento a sus estudiantes, se busca dentro de las instituciones educativas que el trabajo se lo realice en aras de la generación y elaboración de los conocimientos, ciencias y tecnologías propias del país, podemos determinar que el Ecuador, aunque ha trabajado en conseguir implemento tecnológicos para mejora de la educación superior, esta presenta atrasos en el uso adecuado de las TIC y en infraestructura de comunicaciones por parte de profesores y estudiantes, dicha situación que afecta de forma directa al desarrollo productivo nacional y genera un decrecimiento dentro de la creación de puestos de trabajo para los jóvenes profesionales que buscan ingresar al mercado laboral apenas concluido sus estudios universitarios (Vinueza \& Simbaña, 2017).

Las universidades especialmente públicas, viven en constante evaluación en cada una de las carreras que ofertan con la finalidad de fortalecer la calidad de educación y por ende egresar a profesionales competitivos, con capacidad y actitud capaces de desenvolverse en cualquier entorno laboral, siendo la economía como una ciencia social capaz de analizar el comportamiento humano permitiéndole la optimización de recursos puesto que las necesidades son ilimitadas y los recursos escasos; en este contexto sin lugar a duda la carrera de economía con mención en gestión empresarial aborda temas de interés social relevantes capaces de afrontar problemáticas actuales y aportar con soluciones adecuadas, de ello parte la importancia de poseer profesionales correctamente preparados en esta rama, estudiantes inmersos en las ciencias sociales como la carrera de economía se inclinan a la investigación, generación del 
conocimiento, reflexión y formación de los que se especializan en carreras más técnica y profesionales (Ferreira, 2017).

Las TIC en la formación académica proporcionan un desarrollo constante ya que tienen un impacto significativo en la educación, debido a esto surge la necesidad de que se incluyan programas de estudio para que los estudiantes y docentes tengan las competencias y conocimientos necesarios para aprovechar y usar correctamente las TIC (Moguel \& Alonzo, 2009). El perfil del estudiante debe poseer habilidades y competencias que le permitan desarrollarse a ser competitivo en el ámbito laboral, los fundamentos percibidos como transmisores de recursos e información a los estudiantes deben acoplarse a las distintas características individuales de los involucrados y así conseguir una eficaz formación auditiva, visual, multimedia (Cabero, 2015). La investigación se realizó en la carrera de economía con mención en gestión empresarial de la Universidad Técnica de Machala dentro de esta carrera, el estudio de la misma permite adquirir conocimientos plenamente fundamentos en la mayoría de materias, destacándose entre ellas econometría, métodos cuantitativos, finanzas entre otras por lo que el análisis económico se torna importante con el uso de hojas de cálculo, procesadores de datos y distintos programas estadístico informáticos para la construcción teórica y práctica, así como de trabajos de series de tiempo, bases de datos, para estudiar temas relacionados a la producción, generación de excedentes, microeconomía y macroeconomía entre las más principales con temáticas abordadas que comprenden el ámbito laboral, desarrollo, industria e internacional.

En la enseñanza de la economía, los docentes y estudiantes disponen de diversos recursos como el equipamiento informático y los recursos elaborados por los diferentes sectores que permiten la integración de las TIC en el aula. En el contexto educativo las TIC pueden ayudar a los estudiantes de economía a adquirir las capacidades necesarias para ser: competentes para usar las TIC; buscadores, analistas y evaluadores de la información; solucionar problemas y tomar decisiones; ser eficaces y creativos a partir de herramientas de productividad; estar informados, para ser responsables y capaces de contribuir con la sociedad; analizar y realizar proyecciones de información, entre otros. Por lo que investigación permitirá determinar que el uso de las tecnologías de la información y comunicación en la enseñanza aprendizaje específicamente en economía es un tema relevante ya que es necesario adaptarse a distintos cambios tecnológicos actuales; programas en líneas, bases de datos entre otros, los estudiantes tienen un vasto interés en la utilización de estas herramientas puesto que les da facilidad para comprensión en diferentes temas en el área no solo económica, sino también contable, matemática y áreas de interés social.

En definitiva, el conocimiento, uso e interés de las herramientas que brindan las TIC por parte de los estudiantes de la carrera de la economía de la UTMACH se constituye en una las estrategias fundamentales al momento de efectuar una determinada actividad académica; la función que desempeña este tipo de tecnologías en el aprendizaje de dicha carrera influye en la adquisición de los conocimientos netamente estructurados. Así mismo cabe destacar que el aporte brindado a la carrera de economía está sujeto a la importancia ofrecida por cada estudiante, es decir al uso eficiente que se haga acerca de este tipo de tecnologías. 


\section{Materiales y métodos}

La investigación fue de tipo descriptiva y explicativa. La investigación explicativa se orienta a establecer las causas que originan un fenómeno determinado, en este sentido desde la aparición del internet, los recursos disponibles, para los docentes y estudiantes en el proceso de enseñanza - aprendizaje, se han incrementado y el uso de recursos de las TIC se ha generalizado. Con el método deductivo para la selección y discusión de los datos con un análisis cuantitativo de los datos que permitió analizar y describir detalladamente los resultados obtenidos durante el proceso de investigación. Se realizó una observación directa acerca de la forma en que utilizan las tecnologías de información y comunicación los estudiantes de la Universidad Técnica de Machala pertenecientes a la carrera de economía, con corte transversal en periodo académico 2019-D1.

El análisis detallado de cada elemento de estudio permitió deducir que el uso e interés de las TIC es representativo para un gran número de los estudiantes. Así mismo, para el análisis y conteo estadístico de los datos se utilizó las herramientas estadísticas como el SPSS versión 24 (Statistical Package for the Social Sciences), la misma que arrojo resultados como tablas de frecuencia y graficas estadísticas de las diferentes variables empleadas.

\section{Resultados}

Esta investigación permitió conocer como la sociedad ha cambiado y como los recursos educativos se han ido adaptando a estos cambios. En este sentido, el internet supuso una revolución para diversos profesionales como los docentes, debido a que puso a su disposición diversos recursos didácticos para mejorar el proceso de enseñanza - aprendizaje y que éstos se encuentran a la par de la evolución tecnológica, económica y social. Como el uso de las tecnologías de la información y comunicación en el aprendizaje de la universidad ecuatoriana: desarrollado en la institución de educación superior pública Universidad Técnica de Machala en la enseñanza de la carrera de economía es un tema de importancia ya que los métodos de enseñanza-aprendizaje requieren adaptarse a los cambios tecnológicos actuales. La investigación muestra los resultados que están expresados de manera general y poseen un alto nivel de significancia obtenida a través de la investigación, que, de un total de 377 estudiantes correspondientes a la carrera de economía de la UTMACH, se encuesto a 161 de los mismos. El estudio se enfocó en demostrar el uso, percepción e influencia de las TIC en las actividades académicas de los estudiantes, así como: el acceso a los recursos tecnológicos, la apropiación y uso de herramientas informáticas y el rol que estas cumplen.

En la tabla 1 manifiesta que los estudiantes tienen un alto grado de discernimiento y comprensión referente a las TIC debido a que 136 de los mismos, es decir un 84,5\% señalaron una respuesta afirmativa, mientras que 25 de los mismos es decir el 15,5\% restante señalaron no tener conocimiento acerca de este tema. 
Tabla 1

Conocimiento sobre las TIC

\begin{tabular}{llccc}
\hline & & Frecuencia & Porcentaje & Porcentaje válido \\
\hline \multirow{3}{*}{ Válido } & Si & 136 & 84,0 & 84,5 \\
& No & 25 & 15,4 & 15,5 \\
& Total & 161 & 99,4 & 100,0 \\
\hline Perdidos & Sistema & 1 &, 6 & \\
\hline Total & \multicolumn{5}{c}{162} & 100,0 \\
\hline \multicolumn{5}{c}{ Fuente: elaboración propia }
\end{tabular}

En la tabla 2 se evidencia claramente que la mayoría de estudiantes de la carrera de economía de la Universidad Técnica de Machala creen que el uso de las TIC facilitan el proceso de enseñanza-aprendizaje en su campo de estudio, cabe mencionar que actualmente la implementación de estas herramientas en el aula de clase se ha vuelto indispensable, es por esto que el $92 \%$ del total de encuestados señalaron estar de acuerdo con el uso de estas tecnologías, mientras que el $8 \%$ restante mencionaron no estar de acuerdo.

Tabla 2

Uso de las TIC facilitan el proceso de enseñanza-aprendizaje

\begin{tabular}{llccc}
\hline & & Frecuencia & Porcentaje & Porcentaje válido \\
\hline \multirow{3}{*}{ Válido } & $\mathrm{Si}$ & 148 & 91,4 & 91,9 \\
& $\mathrm{No}$ & 13 & 8,0 & 8,1 \\
& Total & 161 & 99,4 & 100,0 \\
\hline Perdidos & Sistema & 1 &, 6 & \\
\hline Total & \multicolumn{5}{c}{162} & 100,0 \\
\hline \multicolumn{5}{c}{ Fuente: elaboración propia }
\end{tabular}

En la tabla 3 se muestra una mayor concentración del 85,2\% de estudiantes que, si posees de acceso a internet fuera de la universidad, por otra parte, un 14,2\% no cuentan con este servicio de internet. Esta situación admite que la universidad debe promover continuamente la mejoría del acceso a internet para los estudiantes debido a que es una herramienta necesaria para la formación profesional.

Tabla 3

Acceso a Internet fuera del campus universitario

\begin{tabular}{|c|c|c|c|c|}
\hline & & Frecuencia & Porcentaje & Porcentaje válido \\
\hline \multirow{3}{*}{ Válido } & $\mathrm{Si}$ & 138 & 85,2 & 85,7 \\
\hline & No & 23 & 14,2 & 14,3 \\
\hline & Total & 161 & 99,4 & 100,0 \\
\hline $\begin{array}{l}\text { Perdid } \\
\text { os }\end{array}$ & $\begin{array}{l}\text { Siste } \\
\text { ma }\end{array}$ & 1 & 6 & \\
\hline Total & & 162 & 100,0 & \\
\hline
\end{tabular}

Fuente: elaboración propia 
Para las horas indicadas al uso de las TIC en la tabla 4 nos muestra que el $72 \%$ de los estudiantes ocupan entre dos y cuatro horas, el $51 \%$ menos de dos horas y que un $37 \%$ más de cuatro horas.

Tabla 4

Horas dedicadas al uso de las TIC

\begin{tabular}{ccccc}
\hline & Frecuencia & Porcentaje & Porcentaje válido \\
& $\begin{array}{c}\text { Menos de } 2 \\
\text { horas }\end{array}$ & 51 & 31,5 & 31,7 \\
Válido & $\begin{array}{c}\text { Entre 2 y } 4 \\
\text { horas }\end{array}$ & 72 & 44,4 & 44,7 \\
& Más de 4 horas & 37 & 22,8 & 23,0 \\
\hline Perdid & Sistema & 1 &, 6 & \\
\hline Total & & 162 & 100,0 & \\
\hline
\end{tabular}

Fuente: elaboración propia

En cuanto a las herramientas de las TIC usadas con mayor frecuencia (tabla 5) muestra distintas y variadas respuestas por parte de los mismos, destacándose entre estas el uso de entorno virtual, blogs, proyector, ordenador y celular con un 26,71\%. De igual manera el uso de entorno virtual, blogs, proyector, ordenador, celular y herramientas de Microsoft office nos muestran un 24,84\%. Adicionalmente, la utilización de ordenador, celular y herramientas de Microsoft office señalan un $14,91 \%$ y a su vez el manejo de entorno virtual, blogs y proyector nos indican un 3,73\% de su uso. Todo esto al examinar el uso de las herramientas de forma conjunta. Mientras que estudiarlas de manera individual nos indican que 4,97\% representa el uso de entorno virtual, 4,97\% indica la utilización de blogs, 1,24\% revela el uso del proyector, 4,97\% manifiestan el manejo del ordenador, 6,83\% usan únicamente el celular y finalmente otro 6,83\% representa la utilización exclusiva de herramientas de Microsoft office. 


\section{Tabla 5}

Herramientas usadas con mayor frecuencia

\begin{tabular}{|c|c|c|c|c|}
\hline & & Frecuencia & Porcentaje & $\begin{array}{l}\text { Porcentaje } \\
\text { válido }\end{array}$ \\
\hline \multirow{6}{*}{$\begin{array}{l}\text { Váli } \\
\text { do }\end{array}$} & $\begin{array}{l}\text { Blogs, Ordenador, Herramientas de Microsoft } \\
\text { Office }\end{array}$ & 22 & 13,66 & 13.7 \\
\hline & $\begin{array}{l}\text { Entorno virtual, celular, Herramientas de Microsoft } \\
\text { Office }\end{array}$ & 26 & 16.15 & 16.2 \\
\hline & $\begin{array}{l}\text { Entorno Virtual, Blogs, Proyector, Ordenador, } \\
\text { Celular, Herramientas de Microsoft Office }\end{array}$ & 40 & 24,7 & 24,8 \\
\hline & Entorno Virtual, Blog, Proyector & 6 & 3,7 & 3,7 \\
\hline & $\begin{array}{l}\text { Ordenador, Celular, Herramientas de Microsoft } \\
\text { Office }\end{array}$ & 24 & 14,8 & 14,9 \\
\hline & $\begin{array}{l}\text { Entorno Virtual, Blogs, Proyector, Ordenador, } \\
\text { Celular }\end{array}$ & 43 & 26,5 & 26,7 \\
\hline Total & & 161 & 99,4 & 100,0 \\
\hline \multirow{2}{*}{\multicolumn{2}{|c|}{ Perdidos Sistema }} & 1 & ,6 & \\
\hline & & 162 & 100,0 & \\
\hline
\end{tabular}

Fuente: elaboración propia

De acuerdo a que si los estudiantes tienen más interés en las actividades tradicionales en la tabla 6 se evidencia que los estudiantes de la carrera de economía tienen un interés normal por las actividades tradicionales para aprender economía con un 49,7\%; mientras que un mínimo de $8,7 \%$ consideran de mucho interés la realización de este tipo de actividades, claramente se denota que los estudiantes tienen una despejada idea de que la implementación de herramientas tecnológicas para el aprendizaje es de importancia para su educación en la carrera. 
Tabla 6

Más Interés a las actividades tradicionales

\begin{tabular}{ccccc}
\hline & & Frecuencia & Porcentaje & Porcentaje válido \\
\hline \multirow{4}{*}{ Válido } & $\begin{array}{c}\text { Muy } \\
\text { poco }\end{array}$ & 20 & 12,3 & 12,4 \\
& Poco & 47 & 29,0 & 29,2 \\
& Normal & 80 & 49,4 & 49,7 \\
& Mucho & 14 & 8,6 & 8,7 \\
& Total & 161 & 99,4 & 100,0 \\
\hline Perdid & Sistema & 1 &, 6 & \\
\hline Total & & 162 & 100,0 & \\
\hline
\end{tabular}

Fuente: elaboración propia

En el aula de clase algunos estudiantes prefieren elegir sus actividades de acuerdo a sus necesidades, la tabla 7 nos muestra que un 54,7\% considera normal este tipo de tarea, no obstante, con un porcentaje mínimo de $10,6 \%$ muy pocos estudiantes prefieren diseñar sus actividades de manera personalizada. El hecho de que los estudiantes elijan sus actividades a efectuar, permite mayor comodidad y concentración en sus tareas.

Tabla 7

Actividades personalizadas

\begin{tabular}{|c|c|c|c|c|}
\hline & & Frecuencia & Porcentaje & Porcentaje válido \\
\hline \multirow{5}{*}{ Válido } & $\begin{array}{l}\text { Muy } \\
\text { poco }\end{array}$ & 17 & 10,5 & 10,6 \\
\hline & Poco & 28 & 17,3 & 17,4 \\
\hline & Normal & 88 & 54,3 & 54,7 \\
\hline & Mucho & 28 & 17,3 & 17,4 \\
\hline & Total & 161 & 99,4 & 100,0 \\
\hline \multirow{2}{*}{$\begin{array}{l}\text { Perdid } \\
\text { os } \\
\end{array}$} & Sistema & 1 & ,6 & \\
\hline & otal & 162 & 100,0 & \\
\hline
\end{tabular}

Fuente: elaboración propia

La tabla 8 nos presenta que el uso de ordenador para la realización de actividades académicas suele ser muy agotador, los resultados muestran claramente que un 42,9\% de los estudiantes tienen un comportamiento normal de cansancio al usar el computador; mientras que mínimo de estudiantes que representa un $9,9 \%$ muy pocas veces resultan agotados. La correcta distribución de horas para la ejecución de tareas en la computadora es importante para un adecuado rendimiento académico. 
Tabla 8

Usar ordenador e internet cansa

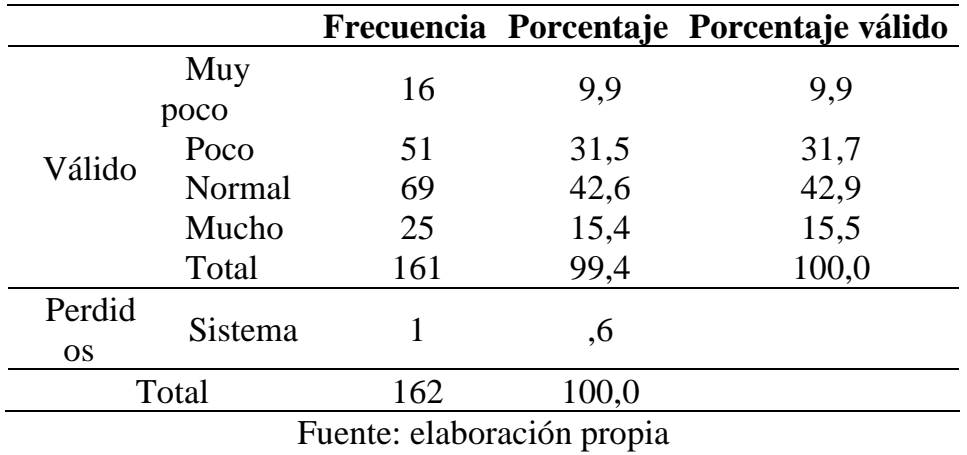

Algunas veces existen ciertos estudiantes que presentan falencias en el empleo de herramientas tecnológicas para su educación en la tabla 9 se muestra que existe un comportamiento normal de ayuda a sus compañeros con un $54 \%$, mientras que con un mínimo porcentaje de 8,7\% muy poco optan por ayudar aquellos compañeros que tienen poco conocimiento en el manejo de estos recursos de tecnología en la carrera de economía de la UTMACH.

Tabla 9 Ayuda compañeros frente al uso de TIC

\begin{tabular}{|c|c|c|c|c|}
\hline & & Frecuencia & Porcentaje & Porcentaje válido \\
\hline \multirow{5}{*}{ Válido } & $\begin{array}{l}\text { Muy } \\
\text { poco }\end{array}$ & 14 & 8,6 & 8,7 \\
\hline & Poco & 26 & 16,0 & 16,1 \\
\hline & Normal & 87 & 53,7 & 54,0 \\
\hline & Mucho & 34 & 21,0 & 21,1 \\
\hline & Total & 161 & 99,4 & 100,0 \\
\hline \multirow{2}{*}{$\begin{array}{c}\text { Perdid } \\
\text { os }\end{array}$} & Sistema & 1 & ,6 & \\
\hline & & 162 & 100,0 & \\
\hline
\end{tabular}

La implementación de las tecnologías de la información y comunicación son importantes para la economía debido a que facilita la búsqueda de información mediante sitios web que ayudan al fortalecimiento de cada estudiante, aportando un grado de significancia a los futuros profesionales, por lo tanto, de las encuestas realizadas a los estudiantes de economía en la tabla 10 presenta que un $88,20 \%$ demostró estar de acuerdo con las TIC en implementarlas en la carrera para la formación de alumnos. 
Tabla 10

De acuerdo con las TIC implementadas en economía

\begin{tabular}{|c|c|c|c|c|}
\hline & & Frecuencia & Porcentaje & Porcentaje válido \\
\hline \multirow{3}{*}{ Válido } & $\mathrm{Si}$ & 142 & 87,7 & 88,2 \\
\hline & No & 19 & 11,7 & 11,8 \\
\hline & Total & 161 & 99,4 & 100,0 \\
\hline $\begin{array}{l}\text { Perdid } \\
\text { os }\end{array}$ & $\begin{array}{l}\text { Siste } \\
\text { ma }\end{array}$ & 1 & 6 & \\
\hline \multicolumn{2}{|c|}{ Total } & 162 & 100,0 & \\
\hline
\end{tabular}

\section{Discusión}

Un hecho innegable en la actualidad es de que la tecnología avanza cada vez más rápido en todos los ámbitos de nuestra sociedad y la educación está entre uno de los más significativos, considerando a los procesos de enseñanza y aprendizaje básicos para la comunicación del estudiante y el colectivo orientado por el docente en procesos de aprendizaje cognitivo en la información (Marqués, 2013). Por lo que se considera que tanto docentes y estudiantes protagonistas del proceso de enseñanza - aprendizaje ven en las tecnologías de la información y comunicación como un recurso didáctico, versátil y poderoso en la educación.

Por lo que existe la necesidad de dar capacitación continua a los profesores con herramientas, cursos, saberes y habilidades en respuestas a los retos que presenta la sociedad y la universidad actual (Jornet, González-Such, \& García-Bellido, 2012), coinciden (Vera, Torres, \& Martínez, 2014) en que la formación y capacitación en tecnología a los docentes debe ser continua formando destrezas competencias tecnológicas y digitales, aplicadas en la formación de los estudiantes, en la que los docentes planifique, asesoren, elaboren, utilicen medios y recursos didácticos dando inicio a la calidad de la formación y actualización en tecnologías de información del conocimiento, por lo que las TIC es aquello de lo que se habla, transmite en relación a su significado, su utilidad, lo que hacen y pueden hacer; el papel que tienen en la educación y en la sociedad, y la descripción que se entrega en torno a su modo de funcionamiento.

El acertado uso de las herramientas de la tecnología de la información y comunicación en el contexto universitario depende del establecimiento educativo, y de los docentes involucrados ya que son los primeros que en visualizar necesidades, oportunidades, ventajas de la institución que pueden ser abarcadas, los docentes deben tener capacitaciones para que comprender de manera fácil y adecuada las distintas herramientas de las TIC educacionales (Márquez, Martínez, Sanjuan, \& Suárez, 2007).

\section{Conclusiones}

El uso de las TIC en la educación resulto como consecuencia del desarrollo del internet y su incursión en los diversos ámbitos de la vida de los individuos. Actualmente la sociedad se desarrolla en un ambiente rodeado de tecnología, por lo que es importante que los docentes y estudiantes se adapten a esta realidad y donde debido a los múltiples recursos que existen, resulta obligatorio la implementación de las TIC dentro del aula y la evaluación de estas en cuanto a los resultados obtenidos en el proceso de enseñanza - aprendizaje. Dentro de las clases de economía 
es frecuente el uso de diversas herramientas como programas estadísticos (Microsoft Excel, Google Spreadsheet, SPSS (SPSS Statistics Base), Minitab, Statgraphics, R The R Project for Statistical Computing, EViews) que permiten un mejor y rápido manejo de la información, programas audiovisuales, acceso a información actualizada,

En definitiva el conocimiento, uso e interés de las herramientas que brindan las tecnologías de la información y comunicación en los estudiantes de la carrera de la economía de la UTMACH se constituye en una las estrategias fundamentales al momento de efectuar una determinada actividad académica; la función que desempeña este tipo de tecnologías en el aprendizaje de dicha carrera influye en la adquisición de los conocimiento netamente estructurados, así mismo es necesario destacar que el aporte ocasionado por las herramientas de tecnología de información y comunicación dentro de la carrera de economía está sujeto a la importancia brindada por cada estudiante, es decir al uso eficiente que se haga acerca de este tipo de tecnologías.

La presencia de las tecnologías de la información y comunicación es primordial para el desarrollo de cada asignatura, ya que el entorno profesional actual exige que los estudiantes no solo adquieran el conocimiento técnico teórico, sino más bien que dominen herramientas tecnológicas como plataformas digitales, software especializado e integración de recursos tecnológicos, por lo que la integración de las tecnologías de la información y comunicación como herramientas tiene que ver con lo que se hace con ellas, tanto en la planificación como en la ejecución y evaluación del proceso de enseñanza-aprendizaje, vinculada a un determinado paradigma de conocimiento y de práctica educativa: a una visión en torno a la forma en que los estudiantes aprenden y en torno a las metodologías que es apropiado utilizar, tanto en la sala de clases, como en instancias educativas formales fuera del aula.

El avance tecnológico evidentemente ha tenido gran impacto en el desarrollo de las actividades académicas, permitiendo a los estudiantes y profesores lograr el objetivo formativo en este sentido, hay que tomar en consideración que las tecnologías de la información y comunicación permiten desarrollar actividades de enseñanza-aprendizaje, más aún que en estos tiempos se encuentra todo tipo de información ya virtualmente ya que tanto como en todos los niveles de estudio empezando desde la niñez hasta ya en formaciones profesionales, ahora adaptándolo en las ciencias económicas nos ha servido de una manera muy sobresaliente ya que se los adapta y fácilmente pueden ser llevados a la práctica y utilizarlos de una manera muy sostenible para mejorar y sean de un aporte efectivo. Con lo que llegamos a concluir que es conveniente el uso de las herramientas de tecnología de la información y comunicación para el desarrollo y aprendizaje en la UTMACH.

\section{Bibliografía}

Altbach, P. (2011). The Past, Present, and Future of the Research University. Economic and Political Weekly, 46(16), 65-73.

Bejar, R. (2007). América Latina, la apuesta tecnológica. Política Exterior, 21(120), 153-161.

Bordas-Beltrán, J., \& Arras-Vota, A. (2018). Perspectivas de los estudiantes mexicanos sobre competencias en TIC, definidas por género. Revista Latina de Comunicación Social, 73, 462-477.

Esta obra se comparte bajo la licencia Creative Common Atribución-No Comercial 4.0 International (CC BY-NC 4.0) Revista de la Universidad Internacional del Ecuador. URL: https://www.uide.edu.ec/ 
Cabero, J. (2015). Reflexiones educativas sobre las tecnologias de la información y la comunicación (TIC). CEF(1), 19-27.

Campos, V., \& Navarro, C. (2017). Participación en redes organizacionales y uso de las tecnologías de la información y la comunicación, un estudio de su impacto en los resultados de la cooperativa de enseñanza de la comunidad valenciana. Revista de Estudios Cooperativos(124), 32-46.

Cárdenas, Y. P. (2012). Herramienta para medir el impacto del nivel de uso y acceso a algunas TIC en el promedio académico de estudiantes de la Facultad de Economía de la Universidad de La Habana. Cuadernos de Educación y Desarrollo, 30.

Duarte, A. (. (2007). Conceptions of Learning and Approaches to Learning in Portuguese Students. Higher Education, 54(6), 781-794.

Escardíbul, J. M. (2016). El efecto de las TIC en la adquisición de competencias. Un análisis por tipo de centro educativo. Revista Española De Pedagogía, 74(264), 317-335.

Fernández-Cruz, F., Fernández-Díaz, M., \& Rodríguez-Mantilla, J. (2018). Diseño y validación de un instrumento de medida del perfil de formación docente en tecnologías de la información y comunicación. Revista Española De Pedagogía, 76(270), 247-270.

Ferreira, A. (2017). Pablo Tavilla. Una carrera de economía para una población desatendida. Cuadernos del CENDES, 34(95), 183-187.

Jornet, J., González-Such, J., \& García-Bellido, M. (2012). La Investigación Evaluativa y las Tecnologías de la Información y la Comunicación (TIC). Revista Española de Pedagogía, 70(251), 93-110.

Lagos, M., \& Silva, J. (2011). Estado de las experiencias 1 a 1 en Iberoamérica. Revista iberoamericana de educación, 56, 75-94.

Lera, F. H., \& Blanco, C. (2003). La Brecha digital un reto para el desarrollo de la sociedad del conocimiento. Revista Económica Mundial, 119-142.

López, B., Cerveró, G., Rodríguez, J., Félix, E., \& Esteban, P. (2013). Learning styles and approaches to learning in excellent and average first-year university students. European Journal of Psychology of Education, 28(4), 1361-1379.

Luna-Romero, Á., Vega, F., \& Carvajal, H. (2018). Formación docente en el uso de las TIC. Revista Universidad, Ciencia y Tecnología(2), 46-52.

Marqués, P. (2013). Impacto de las TIC en la educación: funciones y limitaciones. Revista 3C TIC(3), 1-15.

Márquez, L., Martínez, I., Sanjuan, E., \& Suárez, C. (2007). Efecto De Las TIC Sobre El Comercio Y El Desarrollo Económico. Análisis Para El Caso De España. Estudios de economía aplicada, 1(25), 313-340.

Moguel, S., \& Alonzo, D. (2009). Dimensiones del aprendizaje y el uso de la TIC. el caso de la universidad autónoma de Campeche, México. Revista Iberoamericana de Educación a Distancia, 12(1), 195-211.

Nieto, S., \& Rodriguez, M. (2007). Convergencia de resultados en dos diseños de investigaciòninnovaciòn en enseñanza universitaria a través de las TIC. Revista Española de Pedagogía, 65(236), 27-48.

Ortega, M. (2011). Las nuevas tecnologías como instrumento innovador de la educacion a lo largo de la vida. Revista española de pedagogía, 69(249), 323-338.

Peñaherrera, M. (2011). Evaluación de un Programa de Fortalecimiento del Aprendizaje basado en el uso de las TIC en el contexto Ecuatoriano. Revista Iberoamericana de evaluación educativa, 4(2), 73-91. 
Plaza de la Hoz, J. (2016). Autoridad docente y Nuevas Tecnologías: cambios, retos y oportunidades. Revista Complutense de Educación, 29(1), 269-286.

Quiroga-Parra, D., Torrent-Sellens, J., \& Murcia-Zorrilla, C. (2017). Las tecnologías de la información en América Latina, su incidencia en la productividad: Un análisis comparado con países desarrollados. DYNA, 84(200), 281-290.

Rivero, C., Chávez, A., Vásquez, A., \& Blumen, S. (2016). Las TIC en la formación universitaria. Logros y desafíos para la formación en psicología y educación. Revista de Psicología, 34(1), 186-199.

Ruiz-Jaramillo, J., \& Vargas-Yáñez, A. (2018). La enseñanza de las estructuras en el Grado de Arquitectura. Metodología e innovación docente a través de las TIC . Revista Española de Pedagogía, 76(270), 353-372.

Sánchez, M., \& González, M. (2014). El español y las tecnologías de la comunicación: El español en la Red. Revista Internacional de Lingüística Iberoamericana, 12(24), 135153.

Sefo, K., Granados, J., Lazaro, M., \& Fernández-Larragueta, S. (2017). La formación del profesorado para un uso innovador de las tic: Un estudio de caso en la educación obligatoria en la provincia de Almería. Profesorado Revista de Currículum y Formación del Profesorado, 21(4), 242-258.

Tejedor, F., \& García-Valcárcel, A. (2006). Competencias de los profesores para el uso de las TIC en la enseñanza. Análisis de susconocimientos y actitudes . Revista Española de Pedagogía, 64(233), 21-43.

Unesco. (2013). Enfoques estratégicos sobre las Tics en educación en América Latina y el Caribe. Santiago de Chile: OREALC/UNESCO.

Vera, J., Torres, L., \& Martínez, E. (2014). Evaluación de competencias básicas en tic en docentes de educacion superior en México. Revista de Medios y Educación(44), 143-155.

Vinueza, S., \& Simbaña, V. (2017). Impacto de las TIC en la Educación Superior en el Ecuador. Revista Publicando, 4(11), 355-368.

Vivanco, G. (2015). Educación y tecnologías de la información y la comunicación ¿es posible valorar la diversidad en el marco de la tendencia homogeneizadora? Revista Brasileira de Educação, 20(61), 297-315. 